\title{
Green Leaf Volatiles: Airborne Signals That Protect against Biotic and Abiotic Stresses ${ }^{+}$
}

\author{
Jurgen Engelberth
}

check for

updates

Citation: Engelberth, J. Green Leaf Volatiles: Airborne Signals That Protect against Biotic and Abiotic Stresses. Biol. Life Sci. Forum 2021, 4, 101. https://doi.org/10.3390/ IECPS2020-08634

Academic Editor:

Yoselin Benitez-Alfonso

Published: 1 December 2020

Publisher's Note: MDPI stays neutral with regard to jurisdictional claims in published maps and institutional affiliations.

Copyright: (c) 2020 by the author. Licensee MDPI, Basel, Switzerland. This article is an open access article distributed under the terms and conditions of the Creative Commons Attribution (CC BY) license (https:/ / creativecommons.org/licenses/by/ $4.0 /)$.
Department of Integrative Biology, University of Texas at San Antonio, San Antonio, TX 78249, USA; jurgen.engelberth@utsa.edu; Tel.: +1-210-458-5466

+ Presented at the 1st International Electronic Conference on Plant Science, 1-15 December 2020; Available online: https://iecps2020.sciforum.net/.

\begin{abstract}
Green leaf volatiles (GLVs) are rapidly released by plant leaves upon damage. This makes them ideal signals to convey the presence of a damaging threat to other parts of the same plant, but also to plants nearby. There, GLVs were first found to activate defense responses against insect herbivores and necrotrophic pathogens. Aside from providing direct protection, GLVs also prime those responses resulting in an enhanced and/or accelerated response to these biotic stressors. Recently, it was shown that GLVs also protect against cold stress in plants, resulting in stress-specific transcript accumulation and subsequent reduced damage. This response was further associated with a stimulation of growth after the stress subsided. Common to all those stresses is that they can also cause the release of these compounds. However, the quantities and qualities of the emitted GLVs can vary significantly even in closely related species, suggesting that eco-physiological factors related to biotic and abiotic stresses rather than systematic relationships may have been the driving force for the highly variable emission of these compounds. Still, too little is known about the regulation of GLV emissions, signaling, and responses to support this hypothesis. In this presentation, we will provide an overview of current knowledge regarding biosynthesis and signaling of GLVs in plants and will give an outlook into future areas of research that may provide essential information about the complex biological activities of these compounds.
\end{abstract}

Keywords: green leaf volatiles; insect herbivory; cold stress; biotic stress; abiotic stress; defense; biosynthesis

\section{Introduction}

Green leaf volatiles (GLVs), the typical green smell of plants, are released almost instantly after mechanical damage from oxygenated fatty acids in the chloroplasts [1-3]. There, a lipoxygenase adds molecular oxygen to position 13 of either linolenic or linoleic acid, thereby producing 13-hydroperoxy fatty acids. In the next step, a hydroperoxide lyase (HPL) cleaves of a six-carbon unit, Z-3-hexen-1-al (Z-3-HAL), from 13-hydroperoxy linolenic acid and hexanal from 13-hydroperoxy linoleic acid. Some plants have an isomerase that can convert the Z-3-HAL into its E-2-isoform. All of these aldehydes can further be reduced by an alcohol dehydrogenase to their corresponding alcohols and both Z-3-hexenol (Z-3-HOL) and E-2-hexenol (E-2-HOL) can become esterified to an acetyl, resulting in Z-3-hexen-1-yl acetate (Z-3-HAC) and E-2-hexen-1yl acetate (E-2-HAC). While aldehydes are directly produced in the damaged tissue, the conversion to the alcohols and their esters requires intact cells [4-6].

These compounds were first discovered more than 100 years ago and were mainly considered as metabolic shunts [7]. However, at the end of the last century these compounds were found to induce genes that were typically associated with defense against insect herbivores [8,9]. However, their activity was always considered to be low, and it was questioned whether or not these compounds could play a role in the complex defense machinery of plants. 


\section{Priming of Anti Herbivore Defenses by GLVs}

We investigated the role of GLV in the response of plants against insect herbivores. We used maize (Zea mays) as our model plants. Since direct effects of GLVs on typical antiherbivore defenses appeared to be rather low [8,9], our approach differed from previous ones by not looking into these direct defenses, but rather to investigate the effects of GLVs on subsequent insect herbivory [10]. Plants were therefore exposed overnight with GLVs and then treated the next day with insect-derived elicitors, which were identified previously to mimic insect herbivory [11]. It was found that plants that were previously exposed to GLVs responded stronger and faster to insect herbivory than control plants. Both signaling, which is mainly attributed to jasmonic acid, and responses were significantly increased. Consequently, damage by caterpillars was also significantly reduced in these plants. These results established GLVs as priming agents that prepare plants against impending insect herbivory and have since been confirmed for many other plant species.

To gain further insights into the biological activity of GLVs, we performed a microarray analysis of maize plants that were exposed to GLV [12]. We found that after $20 \mathrm{~min}$, mainly genes involved in general signaling and the regulation of gene expression were significantly increased, whereas after $60 \mathrm{~min}$, genes related to lipid signaling, cell wall modifications, and direct and indirect defenses were also significantly upregulated. Surprisingly, among those genes were also some that were normally considered to be involved in the response to water stress, including dehydrins, proteolipids, low temperature-inducible genes, and others. This prompted us to look further into the potential for GLVs to also regulate responses to abiotic stresses, in particular those related to water stress. Water stress can be caused by many abiotic factors. However, the major problems for plants are drought and low temperatures. We decided to first investigate a potential role of GLVs in the protection of plants against cold stress damage.

\section{Protection against Cold Stress}

While the results from the microarray analysis regarding genes that were involved in the protection of plants against water stress were confirmed by GPCR, we further found that not only were these gene induced by cold stress, but also that priming with GLVs increased the transcript accumulation over the duration of the stress [13]. Consequently, maize seedlings treated with GLVs sustained less damage and were thus better protected. Additionally, we found that GLV and cold-treated maize seedlings showed enhanced growth when moved back to normal conditions. We further established that damage by cold stress primarily caused the release of Z-3-HAL, even when the damage occurred at $-5{ }^{\circ} \mathrm{C}$. This prompted us to ask if GLVs might also protect plants when treated in the cold [14]. We found that even when applied at $4{ }^{\circ} \mathrm{C}$, GLVs would still enhance protective gene expression, reduce damage, and stimulate growth as described above. These studies established a new role for GLV in the protection of plants against cold stress damage.

\section{The Costs of Priming by GLVs against Biotic and Abiotic Stresses}

GLVs increase transcript accumulation for genes involved in defense against insect herbivores, but also genes that play a role in the protection of plants against water stress. This activation of protective genes and consequently, protective proteins and metabolites is usually costly and should result in a reduction in growth and other fitness parameters. To analyze this further, we measured the effect of GLV treatment on the growth of maize seedlings [15]. Since GLVs prime responses against biotic and abiotic stresses, we further analyzed growth responses of GLV-primed plants after cold, mechanical wounding, and treatment with insect elicitors. As already described above, we found a significant effect of GLV priming on the growth response after subsequent cold stress. This was a first indication that GLVs may have a growth stimulating effect rather than just suppressing growth. Additionally, indeed, while over the first $24 \mathrm{~h}$ after GLV treatment a reduction in growth was observed, maize seedlings then began to increase their growth again when compared to controls. Even growth responses of GLV-treated maize seedlings after mechanical 
wounding or treatment with insect elicitor were stronger than in comparable control plants. This growth stimulation was not only observed in the directly treated leaf, but also systemically. These results strongly suggest that GLV-induced priming may have a metabolic component, which still has to be identified and characterized.

\section{The Ecological Relevance of GLVs}

GLVs are considered to be common to all green plants. However, while the biosynthesis of these compounds is generally well described, little is known about how individual plant species use their respective setup of enzymes for the production of specific patterns of GLVs upon damage. To gain further insight into the capacities of different plant species to produce GLVs, we analyzed quantities and qualities of GLVs released from 59 plant species representing 32 families in 23 orders. We found major differences not only in the quantity of those compounds between different plant species, ranging from undetectable levels to almost $100 \mu \mathrm{g}$ per gram of fresh weight, but also in the quality. For example, a clear correlation between the total amount of GLVs and E-2-HAL was identified, suggesting that for maximum GLV production, an isomerase is required. Consequently, a negative correlation between Z-3-HAL and E-2-HAL was also discovered. However, we were not able to determine other principles that govern the capacity to produce GLVs among the selected plants for this study and concluded that ecophysiological considerations, e.g., the abundance and quality of biotic and abiotic stresses, rather than phylogenetic relationships determine the capacity to produce GLVs in nature. The results further suggested that individual approaches based on these data should be used when analyzing the protective effects of GLVs with regard to biotic and abiotic stresses in individual plant species.

\section{Conclusions and Outlook}

To summarize, we have shown that GLVs provide significant protection against biotic stresses including insect herbivory. Moreover, GLVs also provide significant protection against abiotic stresses, including cold, but also drought and heat, all stresses that are directly related to water limitations. As for cold, GLVs are active in protecting against damage caused by this stress even when plants are already under cold stress conditions. However, although they provide significant protection, this does not come at a cost to the plant, as has been shown by the differential effects on growth in GLV-treated maize plants that were exposed to these biotic and abiotic stresses.

We further characterized the distinct emission of damage-induced GLVs in a variety of different plant species and were able to discover previously unknown correlations between the emissions of certain compounds, as well as the overall quantities of GLVs, where the ability to synthesize E-2-HAL is, for example, essential for maximum GLV production. It appears as if ecophysiological pressure rather than phylogenetic relationships regulate GLV production capacities.

This raises the question of how we can make use of the compounds that provide broad protection against a variety of biotic and abiotic stresses, including insect herbivory, necrotrophic pathogen infections, cold stress, drought, and heat, and all this with little effect on growth and development. Approaches to use these compounds to improve crop protection may therefore include alterations in the biosynthesis of GLVs in plants, the treatment of crops with GLVs at regular intervals, as well as improving the perception of GLVs in plants. However, to date, we know very little about how this would actually affect crops in their natural environment. In addition, while we have a good understanding about the biosynthesis of GLVs, very little is known about the signaling processes that are activated by them. It is therefore essential that we continue to investigate these interesting and intriguing compounds and how they affect plants.

Supplementary Materials: The poster presentation is available online at https://www.mdpi.com/ article/10.3390/IECPS2020-08634/s1.

Institutional Review Board Statement: Not applicable. 
Informed Consent Statement: Not applicable.

Data Availability Statement: Not applicable.

Acknowledgments: This research is supported by USDA/NIFA 2018-06371 grant to J.E.

Conflicts of Interest: The author declares no conflict of interest.

\section{References}

1. Hatanaka, A. The biogeneration of green odour by green leaves. Phytochemistry 1993, 34, 1201-1218. [CrossRef]

2. Matsui, K. Green leaf volatiles: Hydroperoxide lyase pathway of oxylipin metabolism. Curr. Opin. Plant Biol. 2006, 9, 274-280. [CrossRef] [PubMed]

3. Matsui, K.; Sugimoto, K.; Mano, J.; Ozawa, R.; Takabayashi, J. Differential metabolism of green leaf volatiles in injured and intact parts of a wounded leaf meet distinct ecophysiological requirements. PLoS ONE 2012, 7, e36433. [CrossRef]

4. Tanaka, T.; Ikeda, A.; Shiojiri, K.; Ozawa, R.; Shiki, K.; Nagai-Kunihiro, N.; Fujita, K.; Sugimoto, K.; Yamato, K.T.; Dohra, H.; et al. Identification of a hexenal reductase that modulates the composition of green leaf volatiles. Plant Physiol. 2018, 178, 552-564. [CrossRef]

5. D'Auria, J.C.; Pichersky, E.; Schaub, A.; Hansel, A.; Gershenzon, J. Characterization of a BAHD acyltransferase responsible for producing the green leaf volatile (Z)-3-hexen-1-yl acetate in Arabidopsis thaliana. Plant J. 2007, 49, 194-207. [CrossRef]

6. Curtius, T.; Franzen, H. Aldehyde aus gruenen Pflanzenteilen. Chem. Zentr. 1911, 2, 1142-1143.

7. Bate, N.J.; Rothstein, S.J. C6-volatiles derived from the lipoxygenase pathway induce a subset of defense-related genes. Plant J. 1998, 16, 561-569. [CrossRef]

8. Arimura, G.; Ozawa, R.; Shimoda, T.; Nishioka, T.; Boland, W.; Takabayashi, J. Herbivory-induced volatiles elicit defense genes in lima bean leaves. Nature 2000, 406, 512-515. [CrossRef]

9. $\quad$ Engelberth, J.; Alborn, H.T.; Schmelz, E.A.; Tumlinson, J.H. Airborne signals prime plants against herbivore attack. Proc. Natl. Acad. Sci. USA 2004, 101, 1781-1785. [CrossRef]

10. Turlings, T.C.J.; Alborn, H.T.; Loughrin, J.H.; Tumlinson, J.H. Volicitin, an elicitor fo maize volatiles in oral secretions of Spodoptera exigua: Isolation and bioactivity. J. Chem. Ecol. 2000, 26, 189-202. [CrossRef]

11. Engelberth, J.; Contreras, C.F.; Dalvi, C.; Li, T.; Engelberth, M. Early Transcriptome Analyses of Z-3-Hexenol- Treated Zea mays Revealed Distinct Transcriptional Networks and Anti-Herbivore Defense Potential of Green Leaf Volatiles. PLoS ONE 2012. [CrossRef] [PubMed]

12. Cofer, T.M.; Engelberth, M.J.; Engelberth, J. Green leaf volatiles protect maize (Zea mays) seedlings against damage from cold stress. Plant Cell Environ. 2018, 41, 1673-1682. [CrossRef]

13. Engelberth, M.; Selman, S.M.; Engelberth, J. In-cold exposure to Z-3-hexenal provides protection against ongoing cold stress in Zea mays. Plants 2019, 8, 165. [CrossRef] [PubMed]

14. Engelberth, J.; Engelberth, M. The cost of green leaf volatile-induced defense priming: Temporal diversity in growth responses to mechanical wounding and insect herbivory. Plants 2019, 8, 23. [CrossRef]

15. Engelberth, J.; Engelberth, M. Variability in the capacity to produce damage-induced aldehyde green leaf volatiles among different plant species provides novel insights into biosynthetic diversity. Plants 2020, 9, 213. [CrossRef] [PubMed] 